\title{
Author Correction: NIN interacts with NLPs to mediate nitrate inhibition of nodulation in Medicago truncatula
}

Jie-shun Lin, Xiaolin Li, Zhenpeng Luo, Kirankumar S. Mysore (D), Jiangqi Wen and Fang Xie (D)

Correction to: Nature Plants https://doi.org/10.1038/s41477-018-0261-3, published online 8 October 2018.

In the version of this Article originally published, the name of author Zhenpeng Luo was incorrectly listed as Zhenpeng Luo Luo. Additionally, nitrate in Fig. $6 \mathrm{~b}$ was incorrectly shown as ' $\mathrm{NO}_{3}{ }^{+}$' rather than ' $\mathrm{NO}_{3}^{-}$'. This has now been amended. 